\title{
On transmission and recovery of three-dimensional image information in optical waveguides*
}

\author{
Amnon Yariv \\ California Institute of Technology, Pasadena, California 91125 \\ (Received 21 August 1975; revised manuscript received 19 December 1975)

\begin{abstract}
The paper considers two questions. The first one is: Is it possible to transmit three-dimensional pictorial information through transparent glass (or other dielectric) fibers? We find that due to modal dispersion, pictorial information is invariably "smeared" in transmission. The second question is: Given nature's reluctance to transmit pictures through fibers, is there anything we can do about it? We suggest that the answer is yes and point to a class of solutions involving nonlinear optical mixing.
\end{abstract}

\section{INTRODUCTION}

It is well known that optical waveguides are capable, in principle, of transmitting pictorial images. ${ }^{1}$ Such guides can consist of a bundle of fibers in which each fiber carries one resolution cell of the picture, or single multimode fibers in which each resolution element is carried on the average by means of one mode of the fiber. The following discussion is limited to this second class of fibers and more specifically to cases where the picture field to be transmitted is coherent and monochromatic.

It is shown that unavoidable phase delays between the waveguide modes cause a loss of the pictorial information but that the process is reversible and that suitable phase compensators can restore the picture. One such compensation based on the use of nonlinear optical mixing is described in this paper. A second holographic approach is described in an accompanying paper. ${ }^{2}$

To keep the discussion from becoming too diffusely general, we will center much of the following treatment on the quadratic index (QI) fiber. ${ }^{3}$ Such fibers seem to be promising candidates for pictorial transmission and the tractability of their mathematical treatment enables us to gain a better understanding of the underlying dispersion problems which lead to loss of pictorial information.

The principle of phase correction which will be considered is general and will apply to any optical waveguide.

\section{QUADRATIC INDEX FIBER}

Here we will consider the problem of transmitting a coherent optical image field through a length $L$ of a dielectric waveguide with an index of refraction profile

$$
n^{2}(x, y)=n^{2}\left[1-\left(n_{2} / n\right)\left(x^{2}+y^{2}\right)\right] \text {. }
$$

We will make use of an extension of the Fresnel diffraction integral to transmission through systems of lenses and mirror ${ }^{4}$ :

$$
\begin{aligned}
f_{1}\left(x_{1}, y_{1}\right)= & \left(\frac{i k}{2 \pi B}\right) e^{-i k L} \iint f_{0}\left(x_{0}, y_{0}\right) \exp \left(-\frac{i k}{2 B}\left[D\left(x_{1}^{2}+y_{1}^{2}\right)\right.\right. \\
& \left.\left.-2 x_{1} x_{0}-2 y_{1} y_{0}+A\left(x_{0}^{2}+y_{0}^{2}\right)\right]\right) d x_{0} d y_{0}
\end{aligned}
$$

where $f_{0}\left(x_{0}, y_{0}\right)$ is the input optical field (i.e., the input picture) and $f_{1}\left(x_{1}, y_{1}\right)$ is the output (transmitted) field in plane 1. The quantities $A, B$, and $D$ appearing in (2) are elements of the ray matrix $(A, B, C, D)$ that relate the output ray $\left(r_{1}, d r_{1} / d z\right)$ to the input ray $\left(r_{0}, d r_{0} / d z\right)$ according to ${ }^{1}$

$$
\left|\begin{array}{c}
r_{1} \\
\frac{d r_{1}}{d z}
\end{array}\right|=\left|\begin{array}{ll}
A & B \\
C & D
\end{array}\right|\left|\begin{array}{c}
r_{0} \\
\frac{d r_{0}}{d z}
\end{array}\right| .
$$

In the case of a composite system the matrix $(A, B, C, D)$ is the product of the individual element matrices.

In our case the planes 1 and 0 are, respectively, the output and input planes of a QI fiber of length $L$. The $A B C D$ matrix relating these two planes is ${ }^{1}$

$$
\begin{aligned}
& A=D=\cos \left[\left(n_{2} / n\right)^{1 / 2} L\right], \\
& B=\left(n / n_{2}\right)^{1 / 2} \sin \left[\left(n_{2} / n\right)^{1 / 2} L\right], \\
& C=-\left(n_{2} / n\right)^{1 / 2} \sin \left[\left(n_{2} / n\right)^{1 / 2} L\right] .
\end{aligned}
$$

It follows from (4) that for fiber lengths $L$ satisfying

$$
\left(n_{2} / n\right)^{1 / 2} L=2 s \pi, \quad s=1,2,3, \ldots
$$

we have

$$
A=D=1, \quad C=B=0 .
$$

The output field $f_{1}\left(x_{1}, y_{1}\right)$ at such planes is obtained by a substitution of ( $5 b)$ into (2). In this limit we can evaluate the integral by the method of stationary phase, obtaining

$$
f_{1}\left(x_{1}, y_{1}\right)=f_{0}\left(x_{1}, y_{1}\right) e^{-i k L},
$$

i. e., the output field is identical to the input field and perfect image transmission obtains. It follows from (2) that at planes $L$ such that $\left(n_{2} / n\right)^{1 / 2} L=\pi\left(2 s+\frac{1}{2}\right)$, the field is the Fourier transform of the input field $f_{0}\left(x_{0}\right.$, $\left.y_{0}\right)$. When $\left(n_{2} / n\right)^{1 / 2} L=(2 s+1) \pi$, an inverted image obtains. Since $f_{0}\left(x_{0}, y_{0}\right)$ may represent the complex field from a three-dimensional object, the output field, likewise, contains all the necessary three-dimensional information.

Unfortunately, relation (2) is not exact. The main mathematical approximation used in deriving it is that of paraxial rays, which approximates the distance along a ray by the projection of the ray along the symmetry axis. ${ }^{5}$ This approximation, which is inherent in much of the formalism of coherent optics ${ }^{6}$ and of the Gaussian beam algebra used in optical resonator theory, ${ }^{7}$ thus leads us to conclude that perfect picture transmission is possible. To test this conclusion, which we suspect is erroneous, we will consider the problem of image transmission in a QI fiber in an essentially exact manner, pausing along the way to note carefully which 
mathematical approximations lead to the conclusion that perfect picture transmission is possible.

We solve Maxwell's propagation equation

$$
\nabla^{2} \mathbf{E}+\omega^{2} \mu \epsilon(\mathbf{r}) \mathbf{E}(\mathbf{r})=-\nabla((1 / \epsilon) \mathbf{E} \cdot \nabla \epsilon) .
$$

For small fractional change of $\epsilon$ per wavelength we can neglect the right-hand side of Eq. (7). We can, in addition, factor out the fast phase variation by defining

$$
E(x, y, z)=\psi(x, y, z) e^{-i k z}, \quad k=(\omega / c) n
$$

and specialize the treatment to the case of a quadratic index variation

$$
\epsilon(r)=\epsilon_{0} n^{2}(r)=\epsilon_{0} n^{2}\left[1-\left(n_{2} / n\right) r^{2}\right], \quad r^{2}=x^{2}+y^{2} .
$$

The wave equation now takes the form

$$
\frac{\partial^{2} \psi}{\partial x^{2}}+\frac{\partial^{2} \psi}{\partial y^{2}}-2 i k \frac{\partial \psi}{\partial z}+\frac{\partial^{2} \psi}{\partial z^{2}}-k^{2} \frac{n_{2}}{n} r^{2} \psi=0 .
$$

The Gaussian beam $A B C D$ formalism widely used in coherent optics and in treating optical resonators ${ }^{1,7}$ is based on assuming slow variation of $\psi$ so that

$$
k \frac{\partial \psi}{\partial z} \gg \frac{\partial^{2} \psi}{\partial z^{2}} \ll k^{2} \psi
$$

which causes (8) to simplify to

$$
\frac{\partial^{2} \psi}{\partial x^{2}}+\frac{\partial^{2} \psi}{\partial y^{2}}-2 i k \frac{\partial \psi}{\partial z}-k^{2} \frac{n_{2}}{n} r^{2} \psi=0 .
$$

The eigenmodes satisfying (10) are

$$
\begin{aligned}
E_{l m}(x, y, z)= & \psi_{l m}(x, y, z) e^{-i \beta_{l m} z} \\
= & H_{l}\left(\frac{\sqrt{2}}{\omega} x\right) H_{m}\left(\frac{\sqrt{2}}{\omega} y\right) \exp \left(\frac{-\left(x^{2}+y^{2}\right)}{\omega^{2}}\right) \\
& \times \exp \left\{-i\left[k-\left(\frac{n_{2}}{n}\right)^{1 / 2}(l+m+1)\right] z\right\},
\end{aligned}
$$

where $H_{l}$ is the Hermite polynomial of order $l$, and $\omega$, the spot size, is given by

$$
\omega=\left(\frac{2}{k}\right)^{1 / 2}\left(\frac{n}{n_{2}}\right)^{1 / 4}=\left(\frac{\lambda}{\pi}\right)^{1 / 2}\left(\frac{1}{n n_{2}}\right)^{1 / 4} .
$$

We will now apply the approximate modes as given by (11) to the problem of image propagation in a QI fiber. An input field $f_{0}\left(x_{0}, y_{0}\right)$ at $z=0$ is expanded in terms of the orthonormal set (11)

$$
f_{0}\left(x_{0}, y_{0}\right)=\sum_{l, m} A_{l m} H_{l}\left(\frac{\sqrt{2}}{\omega} x_{0}\right) H_{m}\left(\frac{\sqrt{2}}{\omega} \cdot y_{0}\right) e^{-\left(x_{0}^{2}+y_{0}^{2}\right) / \omega^{2}},
$$

so that the picture information is specified by means of the complex coefficients $A_{l m}$.

The output field at $z=L$ is obtained by propagating each mode, i.e., by multiplying it by the phase factor

$$
\exp \left(-i \beta_{l m} L\right)=\exp \left\{-i\left[k-\left(n_{2} / n\right)^{1 / 2}(l+m+1)\right] L\right\},
$$

and then summing the modes to obtain

$$
\begin{aligned}
& f_{1}\left(x_{1}, y_{1}\right)=\sum_{l, m} A_{l m} H_{l}\left(\frac{\sqrt{2}}{\omega} x_{1}\right) H_{m}\left(\frac{\sqrt{2}}{\omega} y_{1}\right) \\
& \left.\times e^{-\left(x_{1}^{2}+y\right.} \underset{1}{2}\right) / \omega^{2} \exp \left\{-i\left[k-\left(\frac{n_{2}}{n}\right)^{1 / 2}(l+m+1)\right] L\right\} \text {. }
\end{aligned}
$$

At sets of planes $L$ where the condition

$$
\left(n_{2} / n\right)^{1 / 2} L=2 s \pi, \quad s=1,2,3, \ldots
$$

is satisfied, the phase factors $\exp \left[+i\left(n_{2} / n\right)^{1 / 2}(l+m+1)\right.$ $\times L]$ in (14) are all unity and $f_{1}\left(x_{1}, y_{1}\right)=e^{-i k L} f_{0}\left(x_{1}, y_{1}\right)$, i.e., perfect image transmission results.

We have thus obtained the same result as from the use of the diffraction integral (2). The set of image planes (15) is the same as that obtained in (5a). To test the validity of this conclusion we now solve (8) exactly, i.e., without resorting to the approximation (9). The resulting differential equation is identical to the harmonic oscillator Schrödinger equation, and its eigenmodes $\operatorname{are}^{8}$

$$
\begin{aligned}
E_{l m}(x, y, z)= & H_{l}\left(\frac{\sqrt{2}}{\omega} x\right) H_{m}\left(\frac{\sqrt{2}}{\omega} y\right) \exp \left(\frac{-\left(x^{2}+y^{2}\right)}{\omega^{2}}\right) \\
& \times \exp \left\{-i k\left[1-\frac{2}{k}\left(\frac{n_{2}}{n}\right)^{1 / 2}(l+m+1)\right]^{1 / 2} z\right\} .
\end{aligned}
$$

We note that transverse mode profiles are the same as that of the approximate solution (11). The only difference is in the propagation constant $\beta_{l m}$, which is now given by

$$
\beta_{l m}=k\left[1-(2 / k)\left(n_{2} / n\right)^{1 / 2}(l+m+1)\right]^{1 / 2} .
$$

The propagation constants $\beta_{l m}$ are no longer related by an addition of integral multiples of some constant as in (13). It thus follows that there no longer exist planes $L$ as in (15) where all the modes $l, m$ are in the same phase relationship as in the input plane. The field $f_{1}\left(x_{1}, y_{1}\right)$ at $z=L$ cannot, consequently, be made proportional to the input field $f_{0}\left(x_{0}, y_{0}\right)$ and image information is lost.

By comparing the "exact" solution (16) to the paraxial solution (11) we find that it is indeed the approximation (9) that leads us to conclude, erroneously, that perfect picture transmission is possible. Furthermore, from (16) we find that for distances $L$ such that

$$
\left(\beta_{l m}-\beta_{00}\right) L \ll \pi,
$$

the cumulative phase difference between modes does not spoil the picture reconstruction, and the image information can be extracted. Expanding (17) in powers of $(2 / k)\left(n_{2} / n\right)^{1 / 2}(l+m+1)$ and using the second-order term only [the first-order terms are identical to (13) and thus add up in phase at distances $L$ satisfying (15)], we obtain

$$
L_{\max } \approx \frac{2 \pi^{2}}{\lambda\left(n_{2} / n\right)\left(l_{\max }+m_{\max }+1\right)^{2}}
$$

for the maximum transmission distance. We note that since $l_{\max }$ and $m_{\max }$ are essentially the number of picture resolution elements in the $x$ and $y$ directions, respectively, the distance $L_{\max }$ within which we can recover the picture is smaller the larger the number of resolution elements to be transmitted.

The considerations leading to (19) are of the same physical origin as those used to describe group velocity dispersion and its influence on pulse broadening in optical fibers. ${ }^{9}$ In the latter case, group velocity disper- 
sion manifests itself as a limitation on the number of pulses, i.e., temporal resolution elements, that can be transmitted per second through a length $L$.

It is of interest to recast (19) in a slightly different form which may offer a better physical insight to the number of resolution elements that can be carried a distance $L$ without appreciable distortion. According to (19) we should use a fiber with as small a quadratic coefficient $n_{2}$ as possible. Since the Gaussian spot size $\omega\left[\right.$ see (11)] depends on $n_{2}$ according to

$$
\omega=(\lambda / \pi)^{1 / 2}\left(1 / n n_{2}\right)^{1 / 4},
$$

the minimum value of $n_{2}$ depends on the radius $r_{0}$ of the fiber core. If we choose $n_{2}$ such that $\omega=\frac{1}{2} r_{0}$ and take the total number of resolution elements as $N_{\max }=l_{\max }^{2}$ $=m_{\max }^{2}$, Eq. (19) yields

$$
N_{\max } L_{\max } \approx \pi^{4} n^{2} v_{0}^{4} / 16 \lambda^{3} \text {. }
$$

The advantages of a large radius and a short operating wavelength are apparent.

To gain an appreciation for the magnitudes involved, consider the following typical case:

$$
\begin{aligned}
& l_{\max }, m_{\max }=25 \quad \text { (i.e., } 25 \times 25 \text { resolution elements) } \\
& r_{0}=200 \mu \mathrm{m}, \quad n=1.5, \quad \lambda=1 \mu \mathrm{m} .
\end{aligned}
$$

The maximum usable fiber length is obtained from (20) as $L_{\max } \sim 98 \mathrm{~cm}$.

\section{IMAGE COMPENSATION}

One consequence of treating the problem of image propagation by means of the normal modes of the waveguide as in (14) is that it suggests immediately a method for recovering the image. The following discussion is general and applies to any multimode invariant waveguide with coherent image excitation.

Consider a waveguide with a complete orthonormal set of propagating modes

$$
E_{m n}(x, y) e^{-i \beta} m n^{z} \text {. }
$$

A complex input field [since the input field $f_{0}\left(x_{0}, y_{0}\right)$ is complex the eigenfunctions $E_{m n}(x, y)$ can be considered as complex or, alternatively, we may consider the input field as $f_{0}\left(x_{0}, y_{0}\right)=f_{0 r}\left(x_{0}, y_{0}\right)+i f_{0 i}\left(x_{0}, y_{0}\right)$, where $f_{0 r}$ and $f_{0 i}$ are real functions, and expand each of these by means of a set of real eigenfunctions] $f_{0}(x, y)$ at $z=0$ excites the modes according to

$$
f_{0}\left(x_{0}, y_{0}\right)=\sum_{m, n} A_{m n} E_{m n}\left(x_{0}, y_{0}\right),
$$

where the expansion coefficients $A_{m n}$ are complex. According to (21), the field at some point $x_{0}, y_{0}$ can be visualized as corresponding to the resultant of the addition in the complex plane of a large number of phasors $A_{m n} E_{m n}\left(x_{0}, y_{0}\right)$. At an "output" plane $z=L$ the field becomes, according to (16) and (17),

$$
f_{1}\left(x_{1}, y_{1}\right)=\sum_{m, n} A_{m n} E_{m n}\left(x_{1}, y_{1}\right) e^{-i \beta_{m n} L} .
$$

In obtaining the total field $f_{1}\left(x_{1}, y_{1}\right)$ at a point $\left(x_{0}, y_{0}\right)$ we thus add the same set of phasors $A_{m n} E_{m n}$ in the complex plane except that now each phasor is rotated relative to its original $(z=0)$ position by an angle $-\beta_{m n} L$. If we define the residual phase shift $\Delta_{m n} L$ of mode $(m n)$ by $-\beta_{m n} L=$ integer times $2 \pi+\Delta_{m n} L$, the magnitude and phase of the resultant vector $f_{1}\left(x_{0}, y_{0}\right)$ is different in magnitude and phase from $f_{0}\left(x_{0}, y_{0}\right)$ as soon as $\Delta_{m n} L$ becomes comparable to $2 \pi$ for a considerable fraction of the modes $m, n$ represented in the expansion. Moreover, the ratio $f_{1}\left(x_{0}, y_{0}\right) / f_{0}\left(x_{0}, y_{0}\right)$ becomes a function of position $\left(x_{0}, y_{0}\right)$ corresponding to a loss of image information.

To regain the image information it would be necessary to shift the phase of each mode $m, n$ at $z=L$ by $+\beta_{m n} L$ so that the resulting field is, according to (21),

$$
\begin{aligned}
\left.f_{1} \text { (compensated }\right) & =\sum_{m, n} A_{m n} E_{m n}\left(x_{1}, y_{1}\right) e^{-i\left(\beta_{m n}-\beta_{m n}\right) L} \\
& =f_{0}\left(x_{1}, y_{1}\right) .
\end{aligned}
$$

Holographic means for accomplishing this phase shift will be considered in the accompanying paper. ${ }^{2}$ An alternative method would be to convert the field (22) at $z=L$ into its complex conjugate

$$
\left(f_{1}\right)_{\text {c.c. }}=\sum_{m, n} A_{m n}^{*} E_{m n}^{*} e^{i \beta_{m n} L} .
$$

Possible means for accomplishing this conjugation will be considered below. The conjugate field is allowed to propagate through another section of length $L$. Each mode $m, n$ will exercise according to (20) a phase shift $-\beta_{m n} L$ between $z=L$ and $z=2 L$. Using (22) we thus obtain for the field at $z=2 L$,

$$
f_{2}\left(x_{2}, y_{2}\right)=\sum A_{m n}^{*} E_{m n}^{*},
$$

which is the complex conjugate of input field (20). The original field can be recovered by conventional image inversion or by a second step of field conjugation.

The above method for regaining the image by phase reversal and additional propagation is reminiscent of the spin echoes of magnetic resonance. ${ }^{10}$ The phase $\Delta \beta_{m n} L$ is equivalent to the temporal phase $\Delta \omega t$ accumulated by spins with a local "field" $\left(\omega_{0}+\Delta \omega\right) /|\gamma|$. The field conjugation is equivalent to the $180^{\circ}$ pulse which reverses the order of the precessing spins. The spin echo observed at time $2 t$ is thus equivalent to the image recovery at $z=2 L$.

What is needed is thus a real time method for converting a (complex) optical field into its complex conjugate. One method for performing this operation is by nonlinear optical multiplication as sketched in Fig. 1. A nonlinear crystal is inserted at $z=L$. The signal field at $z=L$ is taken following (22) as

$$
E_{1}=\sum_{m, n} A_{m n} E_{m n}(x, y) e^{i\left(\omega_{1} t-\beta_{m n} z\right)}+\text { c. c. },
$$

where the time dependence $\exp \left(i \omega_{1} t\right)$ has been explicitly introduced. This field "mixes" in the nonlinear crystal with an intense "pump" field at $\omega_{3} \approx 2 \omega_{1}$,

$$
E_{3} e^{i\left(\omega_{3} t-\beta_{3} L\right)}+\text { c. c. }
$$

to yield an output field at $\omega_{2}=\omega_{3}-\omega_{1}$ proportional to the product term ${ }^{8}$ 


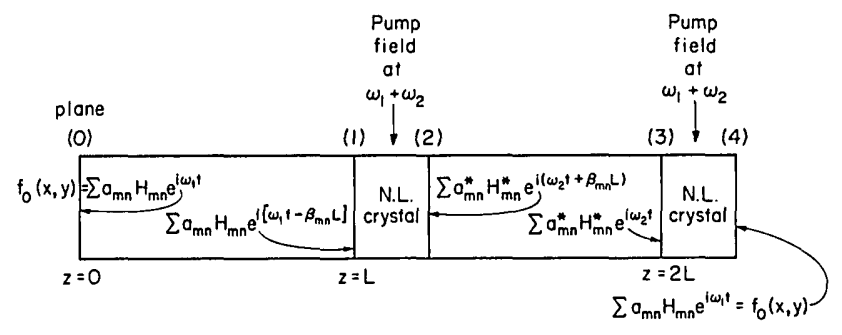

FIG. 1. Multimode optical waveguide with nonlinear crystals used for image restoration. The length of the crystal is assumed very short and is neglected in calculating the modal phase dispersion.

$$
\begin{aligned}
E_{3} E_{1}^{*} & =\sum_{m, n} E_{3} e^{i\left(\omega_{3} t-\beta_{3} L\right)} A_{m n}^{*} E_{m n}^{*} e^{-i\left(\omega_{1} t-\beta_{m n} L\right)} \\
& =E_{3} e^{-i \beta_{3} L} \sum_{m, n} A_{m n}^{*} E_{m n}^{*} e^{i\left(\omega_{2} t+\beta_{m n} L\right)},
\end{aligned}
$$

which is the form of the field at plane 2 of Fig. 1. The derivation of (27) is given in the Appendix.

This field propagates a distance $L$ to plane 3 , where it becomes (assuming $\omega_{2} \approx \omega_{1}$ )

$$
E_{3} e^{-i \beta_{3} L} \sum_{m, n} A_{m n}^{*} E_{m n}^{*} e^{i \omega_{2} t},
$$

and is now mixed again with the pump to give a field proportional to

$$
\sum A_{m n} E_{m n} e^{i \omega_{1} t}=f_{0}(x, y) .
$$

We note that between planes 1 and 2 the field has been converted from frequency $\omega_{1}$ to $\omega_{2}$. It is imperative that before plane 3 , one get rid of the residual field at $\omega_{1}$. One would ideally choose $\omega_{1}=\omega_{2}$ but have the field produced by the nonlinear mixing be orthogonally polarized to the signal field. The mixing described above is well known in the field of nonlinear optics ${ }^{8}$ and can, in principle, be performed so that the resulting beat field at $\omega_{2}$ is more intense than the input field at $\omega_{1}$. This process of parametric amplification may thus be used for image amplification but is not fundamental to the process of phase reversal described above, which may be achieved by other nonlinear means.

We have not considered in this paper problems such as differential mode attenuation, fiber uniformity and intermode scattering. These problems will, in practice, limit the maximum fiber length for which the scheme proposed above will work. They are, however, symptomatic of the imperfect fiber and, as such, subject to continuous improvement. The problem which we addressed above is fundamental and would exist even in the perfect fiber.

In summary, the problem of coherent image transmission in multimode dielectric fibers has been considered. A detailed solution of the propagation problem in a quadratic index fiber shows that within the limits of the conventional paraxial ray treatment one would conclude, erroneously, that perfect image transmission is possible. A more exact treatment reveals the nature of the mode dispersion that leads to loss of pictorial in- formation with distance of propagation. A nonlinear phase reversal technique is discussed which may lead to image recovery. This nonlinear processing of the image in a crystal may be viewed in a general sense as a form of real-time holographic interaction and it is expected that it will find other applications involving image processing and reconstruction.

\section{APPENDIX: MIXING OF THE PICTURE FIELD IN A NONLINEAR OPTICAL CRYSTAL}

In this Appendix we will consider the effect of mixing in a nonlinear crystal of the picture field (22) with the pump laser field, and show how this results in a complex conjugate field as in (27).

From Maxwell's equations

$$
\begin{aligned}
& \nabla \times \overline{\mathbf{E}}=-\mu \frac{\partial \mathrm{H}}{\partial t}, \\
& \nabla \times \mathbf{H}=\frac{\partial}{\partial t}\left(\epsilon_{0} \mathbf{E}+\mathbf{P}\right),
\end{aligned}
$$

we obtain

$$
\nabla^{2} \mathbf{E}=\mu \epsilon_{0} \frac{\partial^{2} \mathbf{E}}{\partial t^{2}}+\mu \frac{\partial^{2}}{\partial t^{2}} \mathbf{P}(r, t)
$$

where $\mathbf{P}=\mathbf{D}-\epsilon_{0} \mathbf{E}$ is the medium polarization. In a nonlinear crystal containing a number of fields at different frequencies we can take the polarization as the sum of a linear term and that due to mixing. We designate the latter as $\mathrm{P}_{\mathrm{NL}}(\mathrm{r}, t)$ and rewrite (A2) as

$$
\nabla^{2} \mathbf{E}-\mu \epsilon(\mathbf{r}) \frac{\partial^{2} \mathbf{E}}{\partial t^{2}}=\frac{\partial^{2}}{\partial t^{2}} \mathbf{P}_{\mathrm{NL}}(\mathbf{r}, t),
$$

where we use $\mathbf{P}=\left(\epsilon-\epsilon_{0}\right) \mathbf{E}$ for the linear polarization.

We now apply (A3) at $z=L$ to the propagation of the picture field (22) within the nonlinear crystal in the presence of a uniform pump field

$$
E_{3}(z, t)=\frac{1}{2} E_{3} e^{i\left(\omega_{3} t-\beta_{3} z\right)}+\text { c. c. }
$$

Taking the picture field frequency as $\omega_{1}$ the effect of nonlinear mixing is to generate a polarization at $\omega_{2}=\omega_{3}$ $-\omega_{1}$. Let us expand the main scalar component of the new field at $\omega_{2}$ as

$$
E_{2}(\mathbf{r}, t)=\frac{1}{2} \sum_{m} B_{m}(z) \mathcal{E}_{2}^{(m)}(x, y) e^{i\left(\omega_{2} t-\beta_{m 2}\right) z} .
$$

The functions $\mathcal{E}_{2}^{(m)}(x, y)$ are the eigenmodes of (A3) with $\mathrm{P}_{\mathrm{NL}}=0$ and thus satisfy

$$
\left(\frac{\partial^{2}}{\partial x^{2}}+\frac{\partial^{2}}{\partial y^{2}}-\beta_{m 2}^{2}\right) \mathcal{E}_{2}^{(m)}+\omega_{2}^{2} \mu \epsilon(\mathbf{r}) \mathcal{E}_{2}^{(m)}=0 .
$$

The subscript 2 refers to the field at $\omega_{2}$, while $B_{m}(z)$ are normal mode amplitudes.

We substitute (A5) into (A3) which, after using (A6), leads to

$$
\begin{gathered}
e^{i \omega_{2} t} \sum_{m}\left[\left(-i \beta_{m 2} \frac{d B_{m}}{d z}+\frac{1}{2} \frac{d^{2} B_{m}}{d z^{2}}\right) \mathcal{E}_{2}^{(m)} e^{-i \beta_{m 2} z}\right]+\mathrm{c} 。 \mathrm{c} . \\
=\mu \frac{\partial^{2}}{\partial t^{2}} P_{\mathrm{NL}}(\mathbf{r}, t)
\end{gathered}
$$

where $P_{N L}(r, t)$ is the component of $\mathbf{P}_{\mathrm{NL}}(\mathbf{r}, t)$ along $\mathbf{E}_{2}$. 
If we assume a "slow" change of $B_{m}(z)$ so that

$$
\left|\frac{d^{2} B_{m}}{d z^{2}}\right| \ll \beta_{m 2}\left|\frac{d B_{m}}{d z}\right|,
$$

we can rewrite (A7) as

$$
\begin{gathered}
\sum_{m}\left(-i \beta_{m 2} \frac{d B_{m}}{d z} \mathcal{E}_{2}^{(m)}(x, y) e^{i\left(\omega_{2} t-\beta_{m} z\right)}\right)+\text { c. c. } \\
=\mu \frac{\partial^{2}}{\partial t^{2}} P_{\mathrm{NL}}(\mathrm{r}, t) .
\end{gathered}
$$

The picture field incident on the nonlinear crystal is taken as

$$
E_{1}(\mathrm{r}, t)=\frac{1}{2} \sum_{l} A_{l}(z) \mathcal{E}_{1}^{(l)}(x, y) e^{i\left(\omega_{1} t-\beta\right.} l 1^{z)}
$$

A comparison of (A9) to (22) establishes the correspondence between the general expansion (A9) and that used in the body of this paper to describe the field in a quadratic index fiber. We choose to keep the general form (A9) since the treatment is independent of the particular waveguide which is used.

The nonlinear polarization at $\omega_{2}=\omega_{3}-\omega_{1}$ is taken as the product of the field at $\omega_{3}[\mathrm{Eq} .(\mathrm{A} 4)]$ and $\omega_{1}$ according to

$$
P_{2}(\mathbf{r}, t)=\sum_{l} \frac{d E_{3} A_{l}^{*}(z)}{2} \mathcal{E}_{1}^{(l)} e^{-i\left(\beta_{3}-\beta_{l 1}\right) z} e^{i\left(\omega_{3}-\omega_{1}\right) t},
$$

where $d$ is the appropriate nonlinear tensor element of the mixing crystal. ${ }^{1}$ Using (A10) in (A8) leads to

$$
\begin{aligned}
\sum_{m} & -i \beta_{m 2} \frac{d B_{m}}{d z} \mathcal{E}_{2}^{(m)} e^{-i \beta_{m 2} z} e^{i \omega_{2} t}+\text { c. c. } \\
& =-\frac{\mu \omega_{2}^{2}}{2} d E_{3} \sum_{l} A_{l}^{*} \mathcal{E}_{1}^{(l)} e^{-i\left(\beta_{3}-\beta_{l 1}\right) z} e^{i \omega_{2} t}+\text { c. c. }
\end{aligned}
$$

We multiply Eq. (A11) by $\mathcal{E}_{2}^{(s)}(x, y)$ and integrate over all $x$ and $y$. We choose the normalization constant of $\mathcal{E}_{2}^{(s)}$ according to

$$
\int_{-\infty}^{\infty} \int \mathcal{E}_{2}^{(s)} \mathcal{E}_{2}^{(m)} d x d y=\delta_{s m} .
$$

The result is

$$
\begin{aligned}
-i \beta_{s 2} \frac{d B_{s}}{d z}= & -\frac{\omega_{2}^{2} \mu}{2} d E_{3} \sum_{l} A_{l}^{*} e^{-i\left(\beta_{3}-\beta_{l 1}-\beta_{s 2}\right) z} \\
& \times \int_{-\infty}^{\infty} \int \mathcal{E}_{1}^{(l)} \mathcal{E}_{2}^{(s)} d x d y .
\end{aligned}
$$

If the frequencies $\omega_{1}$ and $\omega_{2}$ are sufficiently close we can ignore the subscripts in the integral of (A13) and take the orthonormality condition (A13) to apply to a mixed field product, i. e. ,

$$
\int_{-\infty}^{\infty} \int \mathcal{E}_{1}^{(l)} \mathcal{E}_{2}^{(s)} d x d y=\delta_{l s}
$$

which when applied to (A13) results in

$$
\frac{d B_{s}}{d z}=-i \frac{\omega_{2}^{2} \mu}{2 \beta_{s 2}} d E_{3} A_{s}^{*} e^{-i\left(\Delta \beta_{s}\right) z}
$$

and in a like manner,

$$
\frac{d A_{s}^{*}}{d z}=i \frac{\omega_{1}^{2} \mu}{2 \beta_{s 1}} d E_{3} B_{s} e^{i\left(\Delta \beta_{s}\right) z},
$$

where

$$
\Delta \beta_{s} \equiv \beta_{3}-\beta_{s 1}-\beta_{s 2} .
$$

Equations (A15) are of the same form as that of plane wave parametric mixing. ${ }^{1}$ The important new feature here is that in the case of multimode fields, which are considered here, each mode $s$ of the signal $\left(\omega_{1}\right)$ field interacts only with its counterpart $s$ at $\omega_{2}$. This is a manifestation of the need to "phase match" in the transverse direction as well as in the $z$ direction. This lack of mode mixing can be spoiled if $E_{3}$ is not uniform in the $x-y$ plane or if (A14) is not obeyed.

$$
\begin{aligned}
& \frac{d B_{s}}{d z}=-i \frac{g}{2} A_{s}^{*} e^{-i\left(\Delta \beta_{s}\right) z}, \\
& \frac{d A_{s}^{*}}{d z}=i \frac{g}{2} B_{s} e^{i\left(\Delta \beta_{s}\right) z} ; \\
& g \approx \omega(\mu / \epsilon)^{1 / 2} d E_{3} .
\end{aligned}
$$$$
\text { If } \omega_{2} \approx \omega_{1} \equiv \omega \text {, we can rewrite (A15) as }
$$

Referring to Fig. 1 we take the input plane to the nonlinear crystal as $z=L$. The picture field (at $\omega_{1}$ ) is given by (21) so that we associate

$$
A_{s}(0) \rightarrow A_{m n},
$$

and from (22)

$$
A_{s}^{*}(L)=A_{s}^{*}(0) e^{+i \beta_{s 1} L} \text {. }
$$

Equation (A19) is used as a boundary condition in solving (A17). The second condition is $B_{s}(L)=0$ since the modes $B_{s}$ at $\omega_{2}$ are generated by the mixing crystal at $z>L$ and only forward waves are considered. With these conditions the solution of (A17) for $z>L$ is $^{1}$

$$
\begin{aligned}
& B_{s}(z)=-\frac{i g}{2 b} A_{s}^{*}(0) e^{i \beta_{s 1} L} \sinh [b(z-L)] e^{i\left(\Delta \beta_{s} / 2\right)(z-L)}, \\
& b=\frac{1}{2}\left[g^{2}-\left(\Delta \beta_{s}\right)^{2}\right]^{1 / 2}
\end{aligned}
$$

for sufficiently large parametric gain $g \gg \Delta \beta_{s}$ we approximate (A20) by

$$
B_{s}(z)=-i A_{s}^{*}(0) e^{i \beta_{s 1} L} \sinh \left[\frac{1}{2} g(z-L)\right] e^{i\left(\Delta \beta_{s} / 2\right)(z-L)}
$$

The total converted picture field at the output $z=L+l$ of the nonlinear crystal ( $l=$ crystal length) is obtained from (A5) and (A21) as

$$
\begin{aligned}
E_{2}(z=L+l)= & -\frac{i}{2} \sinh \left(\frac{g l}{2}\right) \sum_{m} A_{m}^{*}(0) \mathcal{E}_{2}^{(m)} \\
& \times e^{+i \beta_{m 1} L} e^{i\left(\Delta \beta_{s}\right) l / 2} e^{i \omega_{2} t} .
\end{aligned}
$$

The input field at $\omega_{1}$ at $z=L$ is according to (22),

$$
E_{1}(z=L)=\sum_{m} A_{m}(0) \mathcal{E}_{1}^{(m)} e^{-i \beta_{m 1} L} e^{i \omega_{1} t} .
$$

Apart from the phase factor $\exp \left[\frac{1}{2} i(\Delta \beta)_{s} l\right]$, we find that the complex amplitude of the output field (A22) is proportional to the complex conjugate of the input field $(z=L)$ as given by (A23). This fact was used in Eqs. (26) and (27) to characterize the action of the nonlinear 
crystal. The factor $\sinh \left(\frac{1}{2} g l\right)$ can be made to exceed unity, in which case we have parametric gain, i.e., image intensification. The factor $\exp \left[\frac{1}{2} i\left(\Delta \beta_{s}\right) l\right]$ in $(\mathrm{A} 22)$ is very nearly unity in most cases since $l$, in practice, will probably be limited to a few centimeters. It can easily be shown, however, that a subsequent stage of parametric mixing, which is needed to regain the image, will introduce the complimentary factor $\exp \left[-\frac{1}{2} i\left(\Delta \beta_{s}\right) l\right]$, thus correcting even this small phase deviation. The proportionality of the mixed field (27) to $E_{3}$ results, according to (A18) and (A22), in the small gain limit $g L \ll 1$.

\footnotetext{
*Work supported by the Air Force Office of Scientific Research.
}

${ }^{1}$ A. Yariv, Introduction to Optical Electronics (Holt, Rinehart, and Winston, New York, 1971).

${ }^{2}$ A. Gover, C. P. Lee, and A. Yariv, following paper, J. Opt. Soc. Am. 66, 306 (1976).

${ }^{3} \mathrm{~S}$. Kawakami and J. Nishizawa, IEE E Trans. MTT-16, 814 (1968).

${ }^{4}$ P. Baues, Opt. Electron. 1, 37 (1969).

${ }^{5} \mathrm{M}$. Born and E. Wolf, Principles of Optics, 3rd ed. (Pergamon, New York, 1965).

${ }^{6}$ See, for example, J. W. Goodman, Introduction to Fourier Optics (McGraw-Hill, New York, 1968).

${ }^{7}$ H. Kogelnik, Appl. Opt. 4, 1562 (1965).

${ }^{8}$ A. Yariv, Quantum Electronics, 2nd ed. (Wiley, New York, 1975), p. 120.

${ }^{9}$ D. Marcuse, Bell Syst. Tech. J. 52, 1169 (1973).

${ }^{10}$ E. L. Hahn, Phys. Rev. $80,580(1950)$.

\title{
Direct transmission of pictorial information in multimode optical fibers*
}

\author{
A. Gover, C. P. Lee, and A. Yariv \\ California Institute of Technology, Pasadena, California 91125 \\ (Received 21 August 1975; revised manuscript received 19 December 1975)
}

\begin{abstract}
The problem of coherent image transmission through a single multimode optical fiber is discussed. A scheme is presented for recovering the transmitted image after distortions brought about by the fiber modes dispersion. Realization of this scheme by holographic techniques and with lens systems is proposed, and its limitations pointed out. The application of this scheme in canceling out temporal signal dispersion in a multimode fiber transmission line is also discussed briefly.
\end{abstract}

\section{INTRODUCTION AND QUALITATIVE DESCRIPTION OF THE PROBLEM}

Recent developments in the area of low-loss optical fibers promise to make optical communication via fibers a reality in the near future. Most of the systems considered today, involve some sort of electrical modulation of an optical beam propagating in a fiber. The information is recovered at the receiving end after detection and decoding.

To obtain high data rates (say rates in excess of 200 Mbits/s) in the type of system described above it will be necessary to use single mode fibers in order to avoid pulse spreading or distortion due to modal dispersion. ${ }^{1}$

In this paper we wish to examine the basic problem of direct image transmission in multimode optical fibers by means of coherent light. By direct transmission we mean a system whereby an image, which, in principle, can be three dimensional, is projected onto the input end of a fiber and is recovered in real time at the output end without the need for electronic intermediaries. A second approach to the same problem based on nonlinear mixing is described in an accompanying paper. ${ }^{2}$

Let the multimode optical waveguide, henceforth to be designated as the channel, possess a discrete number $N$ of confined propagating modes of the form

$$
E_{p}(x, y, z)=\epsilon_{p}(x, y) \exp \left(-i \beta_{p} z\right),
$$

where $z$ is the cylindrical axis of the propagation channel, $\epsilon_{p}(x, y)$ is a function describing the transverse field distribution, and $\beta_{p}$ is the propagation constant of the $p$ th mode. The modes $\epsilon_{p}(x, y)$ are orthonormal in the sense

$$
\int_{\text {cross section }} \epsilon_{p}(x, y) \epsilon_{p^{\prime}}(x, y) d x d y=\delta_{p p^{\prime}} .
$$

Now consider a coherent picture field $u(x, y, 0)$ that is projected onto the input $(z=0)$ face of the channel. The field excites the discrete as well as the radiation, i. e., nonconfined, modes of the channel. Since the radiation modes are lost in a short distance and cannot contribute to the output for any reasonable channel length, we take the effective input field as

$$
u_{1}(x, y)=\sum_{p=1}^{N} A_{p} \epsilon_{p}(x, y),
$$

where, using (2),

$$
A_{p}=\int_{\text {cross section }} u(x, y, 0) \epsilon_{p}(x, y) d x d y .
$$

The field $u_{1}(x, y)$ is thus the field that results from spatial frequency band limiting of the true input field $u(x, y, 0)$. It is limited to $\sim N$ resolution elements (where $N$ is the number of confined modes), which is a reflection of the fact that the fiber has a finite numerical aperture. In the following discussion we will consider the problem of transmitting and recovering the field $u_{1}(x, y)$.

To obtain the form of field at the output end $(z=L)$ of the channel we propagate each mode with its characteristic propagation constant and receive from (3)

$$
u_{2}(x, y, L)=\sum_{p=1}^{N} A_{p} \epsilon_{p}(x, y) e^{-\alpha_{p} L} \exp \left(-i \beta_{p} L\right),
$$

where the possibility of intermode scattering has been ignored. A comparison of (5) to (3) shows that picture distortion can be attributed to two mechanisms: (a) the 